Gut, 1976, 17, 953-958

\title{
Suppression of giardiasis during the intestinal phase of trichinosis in the mouse
}

\author{
I. C. ROBERTS-THOMSON 1 , D. I. GROVE, D. P. STEVENS ${ }^{2}$, AND \\ K. S. WARREN \\ From the Divisions of Gastroenterology and Geographic Medicine, Department of Medicine, University \\ Hospitals, Cleveland, Ohio, USA
}

SUMMARY The interaction of the intestinal phases of Giardia muris and Trichinella spiralis was investigated in Swiss albino mice. Intraoesophageal inoculation of G. muris cysts seven days before, or seven days after, similar inoculation of $T$. spiralis larvae resulted in significant reduction in the numbers of Giardia trophozoites in small bowel and Giardia cysts in stools. This effect was not observed when $G$. muris cysts were administered after resolution of the intestinal phase of trichinosis. Giardiasis had no effect on trichinosis as assessed by numbers of adult worms in small bowel and larvae in skeletal muscles. Studies of small bowel morphology showed that the intestinal phase of trichinosis was associated with increased numbers of inflammatory cells in the lamina propria, a significant increase in Paneth cells in crypts, and a marked reduction in the villus:crypt ratio of jejunum. These observations suggest that the intestinal phase of trichinosis induced environmental changes in small bowel, perhaps related to inflammation, which resulted in suppression of proliferation of Giardia trophozoites.

The proliferation of pathogens within the gastrointestinal tract is influenced by the microbial flora (Freter, 1974), intestinal motility (Knop and Rowley, 1975), and local and systemic immunity (Rowley, 1974). Other factors such as the chemical and enzyme composition of the intestinal environment and the morphological characteristics of small bowel may be of importance but remain poorly understood. Examination of simultaneous infection by two intestinal pathogens has provided an approach to the understanding of host defence mechanisms.

The development of a mouse model of giardiasis (Roberts-Thomson et al., 1976) permitted study of the interaction of G. muris and T. spiralis in Swiss albino mice. Giardia muris infection is characterised by proliferation of trophozoites in small bowel and excretion of cysts in stools. Infection resolves spontaneously after 21 to 42 days. $T$. spiralis infection has both an intestinal and a muscle phase (Villela, 1970). The intestinal phase is characterised by the

${ }^{1}$ Melbourne Reciprocal Fellow to University Hospitals of Cleveland.

${ }^{2}$ Address for reprint requests: Dr David P. Stevens, Department of Medicine, University Hospitals, Cleveland, Ohio 44106, USA.

Received for publication 4 August 1976 presence of adult worms in small bowel. The worms release larvae which penetrate the mucosa, pass to skeletal muscle, and mature to third-stage (infective) larvae. Adult worms are eliminated from the small bowel after 10 to 14 days. Both infections induce inflammation of small bowel associated with reduction in the villus:crypt ratio of intestinal mucosa. The interaction of the small bowel phase of each of these infections on the natural history of the other was studied in an effort to clarify non-immunological host defence mechanisms.

\section{Methods}

MICE

Outbred female Swiss albino mice (CF-1), 16 to $20 \mathrm{~g}$ in weight, were obtained from Carworth Farms Inc., New City, New York. All animals were determined to be free of $G$. muris infection before these experiments by the absence of cysts in stools.

ISOLATION AND QUANTITATION OF

G. muris CYSTS

Methods for the isolation and quantitation of Giardia cysts have been described previously (RobertsThomson et al., 1976). Stools from infected mice 
were broken up in tap water, and the faecal suspension was layered on $1 \mathrm{M}$ sucrose (specific gravity $1 \cdot 11$ ) and centrifuged at $400 \mathrm{~g}$ for 15 minutes. Cysts concentrated at the water-sucrose interface were removed, washed in normal saline, and sedimented by centrifugation at $600 \mathrm{~g}$ for 10 minutes. After resuspension in saline, cysts were counted in a haemocytometer chamber, and administered by peroral inoculation into the oesophagus. Quantitation of the subsequent excretion of cysts in stools was performed at weekly intervals. Individual mice were isolated in cages without bedding for two hours. All stools obtained during this period were broken up in $3 \mathrm{ml}$ tap water and isolated on a sucrose gradient as above. Cysts were resuspended in $0.5 \mathrm{ml}$ saline, counted in a haemocytometer chamber, and the number of cysts excreted per two hours was calculated.

QUANTITATION OF G. muris TROPHOZOITES After collection of stools for cysts, mice were killed and the small bowel was removed. A $1 \mathrm{~cm}$ segment of small bowel was excised at a distance of $10 \mathrm{~cm}$ from the gastroduodenal junction for histological study. The remaining two segments were each flushed with $5 \mathrm{ml}$ normal saline, the perfusates pooled, and motile trophozoites counted in a haemocytometer chamber. If trophozoites were not detected, the perfusates were reexamined after five-fold concentration by centrifugation at $50 \mathrm{~g}$ for five minutes and resuspension in $2 \mathrm{ml}$ saline.

ISOLATION AND QUANTITATION OF $T$. spiralis LARVAE AND ADULT WORMS

Trichinella larvae were isolated from infected mice by acid-pepsin digestion of whole carcasses (Larsh and Kent, 1949) and administered in $0.2 \mathrm{ml}$ normal saline by peroral inoculation into the oesophagus. Adult worms in the small bowel were quantitated after removal of small bowel mucosa by incubation with $0.04 \%$ sodium hydroxide for 12 hours at $4^{\circ} \mathrm{C}$ (Campbell, 1967). Total carcass larval counts were performed after acid-pepsin digestion of carcasses (Larsh and Kent, 1949).

\section{SMALL BOWEL MORPHOLOGY}

The segment excised for histological study was opened by longitudinal incision, carefully oriented on filter paper, and fixed in $10 \%$ formalin. After routine processing, biopsies were stained with haematoxylin and eosin. The villus:crypt ratio of jejunal mucosa was determined by measurement of villus and crypt heights in 10 representative, well-oriented villi in at least two separate portions of each biopsy (RobertsThomson et al., 1976). Paneth cells were counted in 10 consecutive, well-oriented crypts.

\section{STATISTICAL METHODS}

Giardia cyst counts in stools and trophozoite counts in small bowel were expressed as the geometric mean and differences between groups were analysed by Student's $t$ test. Worm burdens in small bowel and larval counts in muscles were analysed by Student's $t$ test. A mean value for the villus:crypt ratio of small bowel was determined for each mouse and differences between groups were determined by analysis of variance. Paneth cell counts were analysed by Student's $t$ test.

\section{Results}

\section{T. spiralis INFECTION FOLLOWED BY G. muris} INFECTION

To determine the effect of intestinal trichinosis on the natural history of giardiasis, mice were randomly allocated to two groups on day 0 and inoculated with 0 or $300 T$. spiralis larvae. On day 7, all mice were inoculated with $1000 \mathrm{G}$. muris cysts. On days 14,21 , 28 , and 35 , Giardia cyst excretion in stools and trophozoite counts in small bowel were determined in eight mice from each group. Cyst and trophozoite counts were markedly reduced in the group inoculated with Trichinella larvae (Fig. 1). For both cysts and trophozoites, significant differences between control and Trichinella-infected mice occurred on day $14(\mathrm{P}<0.01)$, day $21(\mathrm{P}<0.001)$, and day 28 $(\mathrm{P}<0.02)$ but not on day 35 .

To determine whether suppression of giardiasis was long lasting and if it occurred after resolution of the intestinal phase of trichinosis, mice were inoculated with 1000 Giardia cysts 10 weeks after inoculation with 0 or 300 Trichinella larvae. Ten mice were included in each group. Cyst excretion in stools was determined on days $7,14,21,28$, and 35 after cyst inoculation. There were no significant differences between control and Trichinella-infected mice (Fig. 2).

\section{G. muris INFECTION FOLLOWED BY $T$. spiralis INFECTION}

To determine the effect of giardiasis on the natural history of trichinosis, mice were inoculated on day 0 with 1000 G. muris cysts. On day 7, Giardia infection was confirmed by the presence of cysts in stools, and mice were randomly allocated to two groups and inoculated with 0 or 300 Trichinella larvae. A third group was inoculated with 300 Trichinella larvae without prior inoculation of Giardia cysts. On days $14,21,28$, and 35 , eight mice from each group were studied to determine cyst excretion in stools and trophozoite counts in smal' bowel. In mice inoculated with $T$. spiralis, worm burdens were determined on 

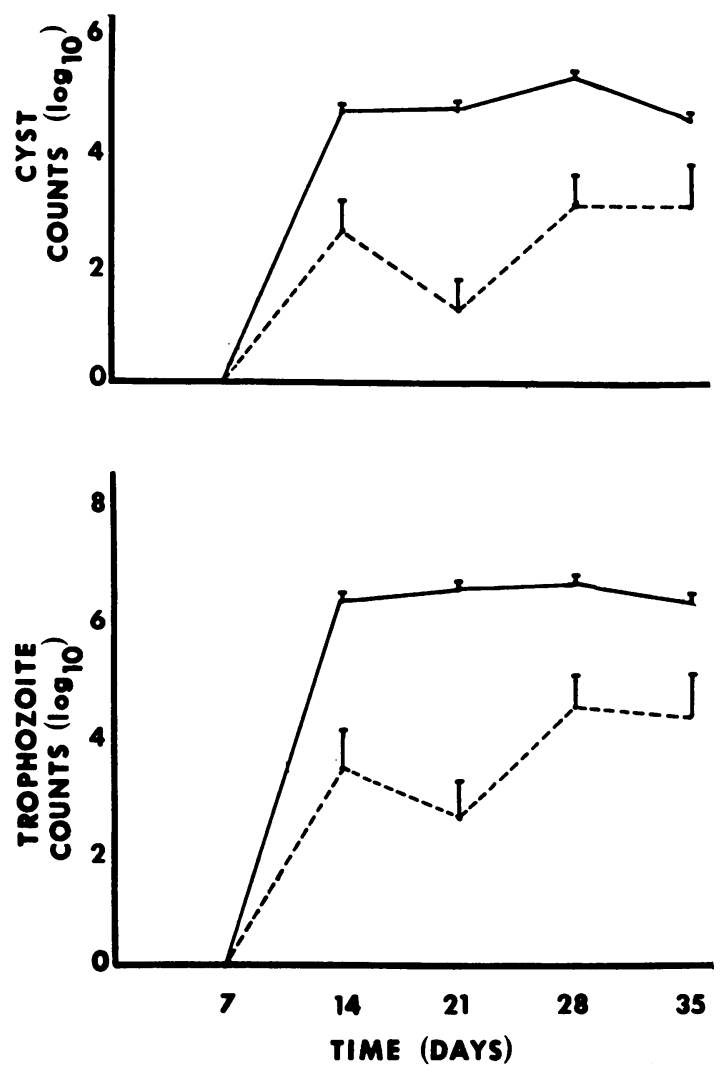

Fig. 1 Giardia cyst counts in stool and trophozoite counts in small bowel after inoculation of 1000 Giardia muris cysts in mice previously infected with 300 Trichinella spiralis larvae (- $\left.-{ }_{-}\right)$or control mice not previously infected with Trichinella ($S E)$. Trichinella larvae were inoculated on day 0 and Giardia cysts were inoculated on day 7.

days 14,21 , and 28 , and carcass larval counts were performed on day 35 .

Worm burdens and carcass larval counts did not differ significantly in mice with and without prior Giardia infection. On days 14, 21, and 28 mean worm counts $( \pm S E)$ in mice infected with both trichinosis and giardiasis $(117 \pm 12,38 \pm 16$, and $1 \pm 1$, respectively) were similar to those in mice infected with trichinosis alone $(159 \pm 17,42 \pm 11$, and $3 \pm 1$, respectively). On day 35 , mean larval counts per carcass $( \pm$ SE) in mice infected with both trichinosis and giardiasis $(18,250 \pm 3,430)$ were similar to those in mice infected with trichinosis alone $(22,140 \pm$ 3,070 ). However, $T$. spiralis infection initiated after established Giardia infection still resulted in suppression of Giardia cyst and trophozoite counts. On day 14 the logarithmic means $( \pm$ SE) of cyst and trophozoite counts in Trichinella-infected mice $(4.5 \pm 0.2$

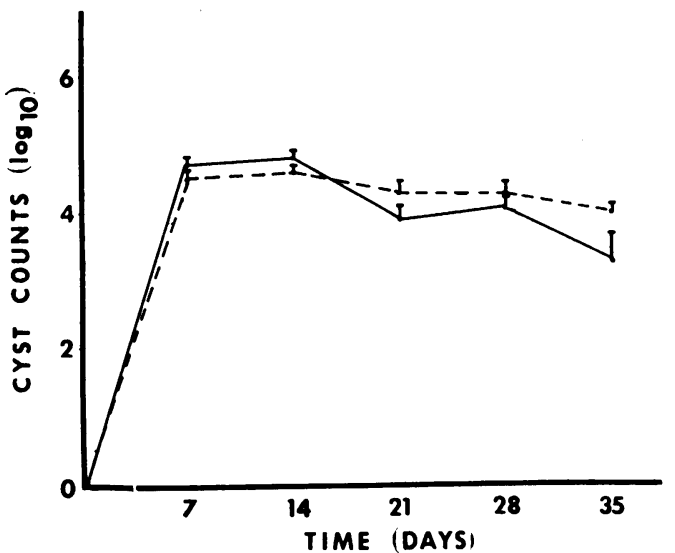

Fig. 2 Giardia cyst counts in stool after inoculation of 1000 Giardia muris cysts in mice in which the intestinal phase of Trichinella spiralis has resolved (- $\left.-{ }_{-}\right)$or in control mice (- $\longrightarrow$ (mean $\pm S E)$. Inoculation of 300 Trichinella larvae was given 70 days before inoculation of Giardia.

and $6.1 \pm 0.3$ respectively) were significantly lower $(P<0.02)$ than those in controls $(5.2 \pm 0.1$ and $6.9 \pm 0.1$ respectively). On day 21 values for Trichinella-infected mice $(2.3 \pm 0.7$ and $6.0 \pm 0.1$ respectively) were again significantly lower $(\mathrm{P}<0.01)$ than in controls $(4.8 \pm 0.1$ and $6.7 \pm 0.1$ respectively). On days 28 and 35 differences between the two groups were not statistically significant.

\section{SMALL BOWEL MORPHOLOGY}

Small bowel morphology was studied in the jejunum, the site of maximal small bowel changes in both giardiasis and trichinosis. Jejunal biopsies were taken from mice killed during the experiment with G. muris as the initial infection. Changes in the villus:crypt ratios are shown in Fig. 3. The mean villus :crypt ratio $( \pm S E)$ in 16 uninfected controls was $3 \cdot 1 \pm 0 \cdot 1$. Giardiasis alone caused a mild but significant reduction in villus:crypt ratio as described previously (Roberts-Thomson et al., 1976). Trichinosis alone caused a marked reduction $(P<0.001)$ in the mean villus:crypt ratio to $1.4 \pm 0 \cdot 1$ on day $14,1 \cdot 0 \pm 0 \cdot 1$ on day 21 , and $1.5 \pm 0.2$ on day 28 . Infection with both Trichinella and Giardia caused a similar pattern of change in the villus:crypt ratio to that induced by Trichinella alone, except that on day 35 the former group showed significant $(P<0.01)$ delay of recovery.

In addition to alteration in the villus:crypt ratio, mice infected with trichinosis showed increased numbers of goblet cells, increased numbers of eosinophils, lymphocytes, and polymorphonuclear leucocytes in the lamina propria, and increased numbers of 

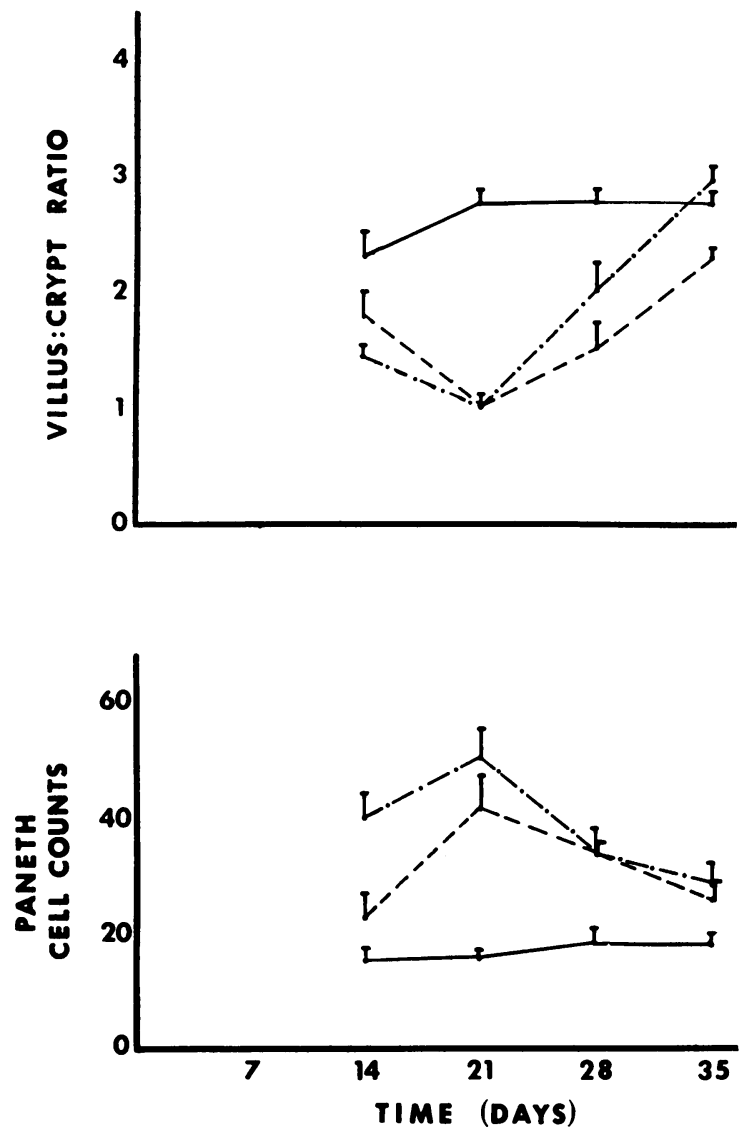

Fig. 3 Changes in the villus:crypt ratio of jejunum, and Paneth cell counts in jejunal crypts in mice with giardiasis alone (-), giardiasis and trichinosis $\left(--_{-}\right)$, and trichinosis alone (----) (mean $\left.\pm S E\right)$. One thousand Giardia muris cysts were inoculated on day 0 and 300 Trichinella spiralis larvae were administered on day 7. Uninfected control mice had a mean villus: crypt ratio of $3.1 \pm 0.1$ and a mean Paneth cell count of $15 \pm 1$.

Paneth cells in crypts (Fig. 4). The mean Paneth cell count $( \pm$ SE) in jejunal biopsies in 16 uninfected controls was $15 \pm 1$. Paneth cell counts did not increase in mice with giardiasis alone but increased significantly on all study days in mice with trichinosis alone, or trichinosis and giardiasis (Fig. 3).

\section{DOSE-RESPONSE STUDIES}

A further experiment was designed to determine whether suppression of giardiasis by trichinosis could be overcome by reducing the Trichinella worm burden. On day 0 , mice were randomly allocated to three groups (eight mice per group) and inoculated with 0,30 , or 300 Trichinella larvae. On day 7 , mice within each group were inoculated with $1000 \mathrm{G}$. muris cysts. On day 14, all mice were studied to determine cyst excretion in stools, trophozoite counts in small bowel, and alteration in the villus:crypt ratio of jejunal mucosa.

The results are shown in Fig. 5. When compared with control mice, animals inoculated with 30 Trichinella larvae showed moderate suppression of cyst $(\mathrm{P}<0.02)$ and trophozoite $(\mathrm{P}<0.05)$ counts and significant reduction in the villus:crypt ratio of jejunum $(P<0.01)$. Marked reduction $(P<0.001)$ in these three parameters was shown in mice infected with 300 Trichinella larvae.

\section{Discussion}

Study of the interaction of G. muris and T. spiralis in Swiss albino mice showed marked suppression of giardiasis by the intestinal phase of trichinosis. This effect was observed regardless of which infection was initiated first. Dose-response studies indicated that the degree of suppression of giardiasis was related to the dose of Trichinella larvae and the extent of reduction in the villus:crypt ratio of small bowel. After resolution of the intestinal phase of $T$. spiralis, resistance to G. muris infection was lost. In contrast, an established $G$. muris infection had no effect on trichinosis as assessed by numbers of adult worms in small bowel and larvae in skeletal muscles.

These observations suggest that Trichinella in small bowel induce intestinal environmental changes which result in suppression of proliferation of Giardia trophozoites. These changes might result from competition for essential nutrients, secretion of toxic products, changes in small bowel morphology, or changes in other characteristics such as intestinal motility, $\mathrm{pH}$, or the concentration of digestive enzymes.

Of particular interest were the observed changes in small bowel morphology. Maximal suppression of giardiasis coincided with maximal reduction in the villus :crypt ratio of small bowel. Reduction in villous height decreases the surface area of jejunal mucosa and might decrease the number of sites for attachment of trophozoites to surface epithelial cells (Erlandsen and Chase, 1974). Alternatively, reduction in villous height might decrease the protective effect of normal villous architecture and make trophozoites more susceptible to intestinal peristalsis. Another possibility is that small bowel inflammation perhaps mediated by thymus-dependent lymphocytes (Walls $e t$ al., 1973), might result in changes in the chemical or enzymic composition of the environment inhabited by the pathogen such that trophozoite proliferation is inhibited.

In trichinosis small bowel inflammation was 


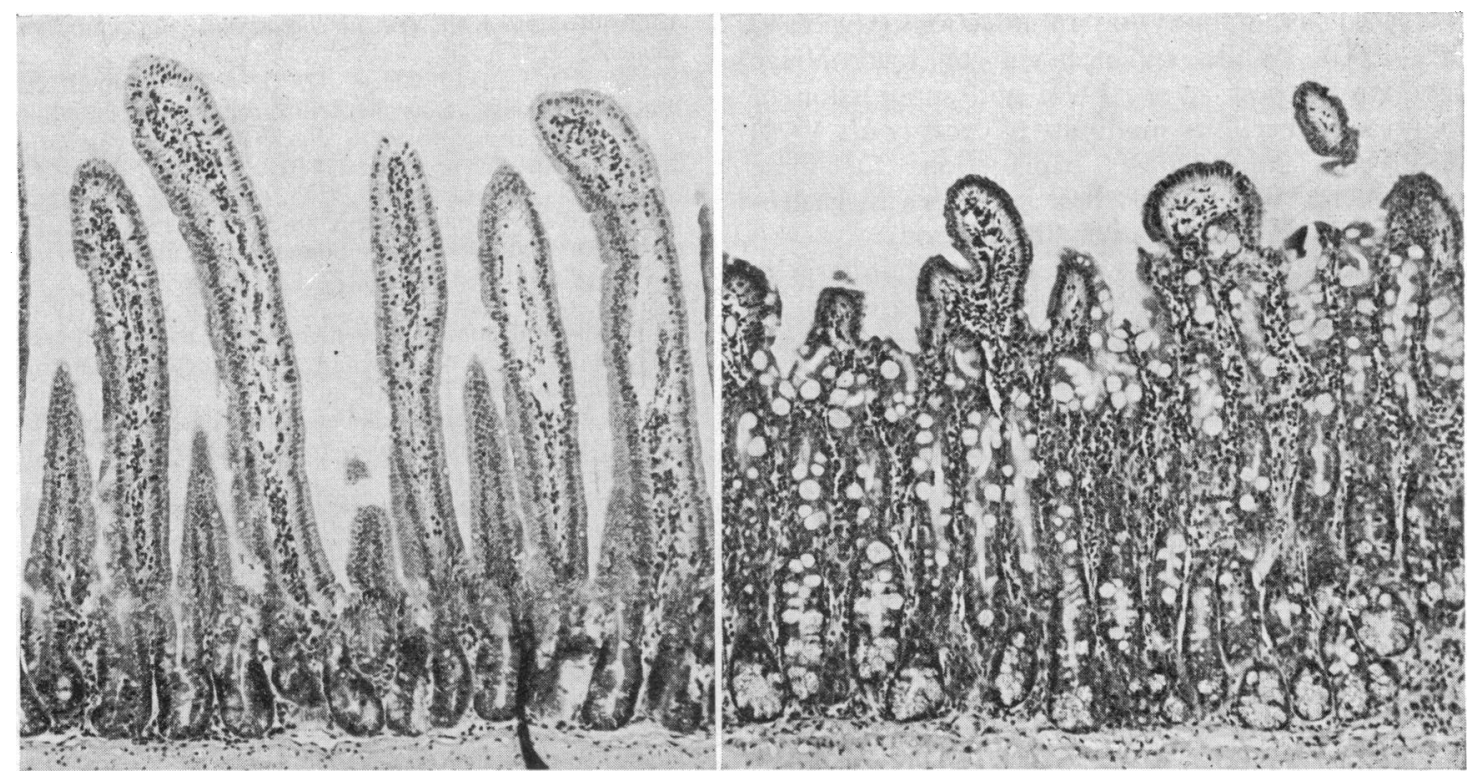

Fig. 4 Jejunal morphology in representative sections from a control (left) and Trichinella-infected (right) mouse inoculated 14 days previously with 300 Trichinella spiralis larvae. Infection with T. spiralis is associated with marked reduction of the villus:crypt ratio. Haematoxylin and eosin, $\times 100$.
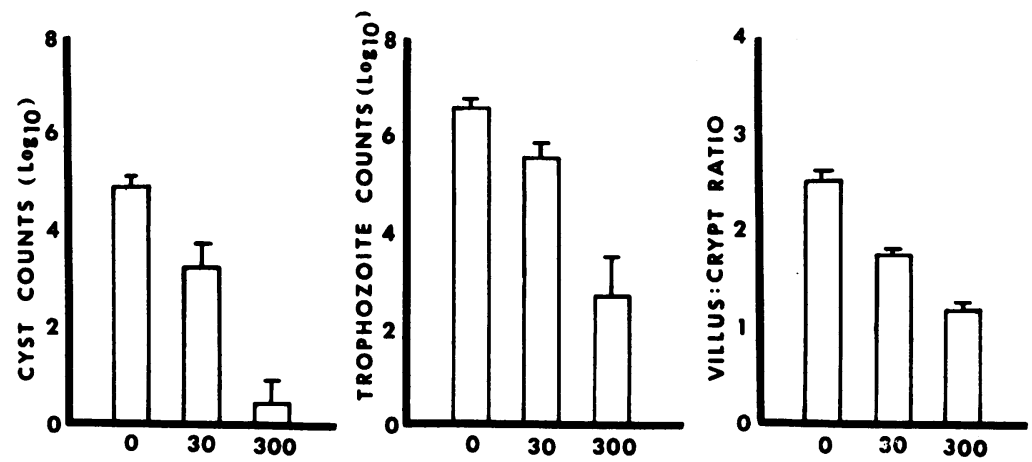

Fig. 5 Giardia cyst counts in stools, trophozoite counts in small bowel, and the villus:crypt ratio of jejunum 14 days after inoculation of 0,30 , and 300 Trichinella spiralis larvae and seven days after inoculation of 1000 Giardia muris cysts (mean $\pm S E)$.

associated with increased numbers of Paneth cells in crypts. These cells arise from undifferentiated crypt cells (Troughton and Trier, 1969) and have secretory granules containing lysosomal enzymes (Riecken and Pearse, 1966), and lysozyme (Erlandsen et al., 1974). In addition, the demonstration of trophozoites of Hexamita muris in Paneth cells in rats (Erlandsen and Chase, 1972) suggests that these cells can function as fixed phagocytes. While increased numbers of Paneth cells might simply reflect increased cell proliferation in crypts, the possibility that trichinosis enhances independent differentiation of Paneth cells cannot be excluded. The absence of increased numbers of Paneth cells in giardiasis does not illuminate this issue, as mucosal lesions were less severe and might be associated with a slower rate of cell proliferation.

The relationship of these observations to reports of other interactions between $T$. spiralis and bacteria, viruses and parasites is unknown. In studies in rodents, infection with $T$. spiralis enhanced survival after intravenous challenge with Listeria monocytogenes (Cypess et al., 1974), decreased parasitaemia after intraperitoneal challenge with Trypanosoma species (Meerovitch and Ackerman, 1974), and 
increased susceptibility to viral infections (Cypess et al., 1973). While enhancement of macrophage function (Cypess et al., 1974) and suppression of humoral and cellular immunity (Cypess et al., 1973) have been suggested as explanations for these phenomena, the role of these processes in gastrointestinal infections is poorly understood.

Our demonstration of suppression of giardiasis by the intestinal phase of trichinosis raises the possibility that important interactions might be found between other gastrointestinal pathogens. In addition, the association of maximal suppression of giardiasis with maximal alteration in small bowel morphology suggests that the proliferation of pathogens might be influenced by factor(s) associated with small bowel inflammation. That the mechanisms are unrelated to immunological cross-reactivity between the parasites is suggested by the absence of suppression of Giardia after resolution of the intestinal phase of trichinosis, and the demonstration of marked suppression of Giardia within seven days of administration of Trichinella larvae.

We thank Miss D. Frank and Mrs E. Moss for expert technical assistance.

\section{References}

Campbell, W. C. (1967). Distribution of Trichinella spiralis in the small intestine of young mice. Journal of Parasitology, 53, 395-397.

Cypess, R. H., Lubiniecki, A. S., and Hammon, W. McD. (1973). Immunosuppression and increased susceptibility to Japanese B encephalitis virus in Trichinella spiralisinfected mice. Proceedings of the Society for Experimental Biology and Medicine, 143, 469-473.

Cypess, R. H., Lubiniecki, A. S., and Swidwa, D. M. (1974). Decreased susceptibility to Listeria monocytogenes in mice after infection with Trinchinella spiralis. Infection and Immunity, 9, 477-479.

Erlandsen, S. L., and Chase, D. G. (1972). Paneth cell function: Phagocytosis and intracellular digestion of intestinal microorganisms. 1. Hexamita muris. Journal of Ultrastructure Research, 41, 296-318.

Erlandsen, S. L., and Chase, D. G. (1974). Morphological alterations in the microvillous border of villous epithelial cells produced by intestinal microorganisms. American Journal of Clinical Nutrition, 27, 1277-1286.

Erlandsen, S. L., Parsons, J. A., and Taylor, T. D. (1974). Ultrastructural immunocytochemical localisation of lysozyme in the paneth cells of man. Journal of Histochemistry and Cytochemistry, 22, 401-413.

Freter, R. (1974). Interactions between mechanisms controlling the intestinal microflora. American Journal of Clinical Nutrition, 27, 1409-1416.

Knop, J., and Rowley, D. (1975). Antibacterial mechanisms in the intestine. Elimination of $V$. cholerae from the gastrointestinal tract of adult mice. Australian Journal of Experimental Biology and Medical Science, 53, 137-146.

Larsh, J. E., and Kent, D. E. (1949). The effect of alcohol on natural and acquired immunity of mice to infection with Trichinella spiralis. Journal of Parasitology, 35, 45-53.

Meerovitch, E., and Ackerman, S. J. (1974). Trypanosomiasis in rats with trichinosis. Transactions of the Royal Society of Tropical Medicine and Hygiene, 68, 417.

Riecken, E. O., and Pearse, A. G. E. (1966). Histochemical study on the Paneth cell in the rat. Gut, 7, 86-93.

Roberts-Thomson, I. C., Stevens, D. P., Mahmoud, A. A. F., and Warren, K. S. (1976). Giardiasis in the mouse: an animal model. Gastroenterology, 71, 57-61.

Rowley, D. (1974). Specific immune antibacterial mechanisms in the intestines of mice. American Journal of Clinical Nutrition, 27, 1417-1423.

Troughton, W. D., and Trier, J. S. (1969). Paneth and goblet cell renewal in mouse duodenal crypts. Journal of Cell Biology, 41, 251-268.

Villela, J. B. (1970). Life cycle and morphology. In Trichinosis in Man and Animals, pp. 19-60. Edited by S. E. Gould. Thomas: Springfield, Illinois.

Walls, R. S., Carter, R. L., Leuchars, E., and Davies, A. J. S. (1973). The immunopathology of trichiniasis in T-cell deficient mice. Clinical and Experimental Immunology, 13, 231-242. 